MS11 Hybrid approaches and validation (X-ray and electron microscopy)

Chairs: Felix Rey, Mathieu Botte

\section{MS11-01 How reliable are atomic models based on cryo-EM reconstructions? Improvements in model fitting and validation.}

\author{
Piotr Neumann ${ }^{1}$, Niels Fischer ${ }^{2}$, Holger Stark ${ }^{2}$, Ralf Ficner ${ }^{1}$
}

1. Department of Molecular Structural Biology, Institute for Microbiology \& Genetics GZMB, Georg-August-Universität Göttingen, Justus-von-Liebig-Weg 11, 37077 Göttingen, Germany 2. 3D Electron Cryomicroscopy Group, Max-Planck-Institute for Biophysical Chemistry, Am Fassberg 11, 37077 Göttingen, Germany

email: pneuman2@uni-goettingen.de

Recent developments in the field of single particle electron cryo-microscopy (cryo-EM) made it possible to obtain 3D reconstruction based atomic models with the overall quality comparable to X-ray crystal structures. However in comparison to X-ray crystallography, the level of structural details is often less uniformly spread across the entire cryo-EM reconstruction, what causes the major difficulty in obtaining reliable atomic models for not well defined or flexible regions. This raises the need of developing new tools for "multi-resolution" fitting of atomic models and their validation or customization of the usage of existing programs.

Our analysis of local Real Space Correlation Coefficient (RSCC) of over 50 near-atomic resolution structures deposited in the Electron Microscopy Data Bank (EMDB) revealed that only a few of those fit the deposited cryo-EM map at a level commonly accepted for $\mathrm{X}$-ray crystal structures (Figure 1). Among the best fitting atomic models is the structure of $70 \mathrm{~S}$ ribosome from Escherichia coli determined at 2.65-2.9 $\AA$ resolution (PDB ID: $5 \mathrm{AFI}^{[\wedge]}$ ) obtained by cryo-EM reconstruction and a pseudo-crystallographic refinement approach employing manual model adjustments, density-guided modeling and energy optimization using the Rosetta package and efficient conformational sampling with the usage of customized "distance-slacked chemical" restraints. Testing the newly established refinement protocol employing customized chemical restraints on the set of analyzed cryo-EM based atomic models will be discussed. Interestingly, re-refinement of analyzed structures revealed significant discrepancies in the highest resolution limit determined by cryo-EM gold-standard FSC and X-ray crystallography derived criteria indicating the need of employing validation techniques assessing to which resolution limit reliable structural features extend.
${ }^{[\wedge}$ Niels Fischer, Piotr Neumann, Andrey L. Konevega, Lars V. Bock, Ralf Ficner, Marina V. Rodnina \& Holger Stark, Structure of the E. coli ribosome-EF-Tu complex at $<3 \AA$ resolution by Cs-corrected cryo-EM, (2015), Nature 520, 567-570.

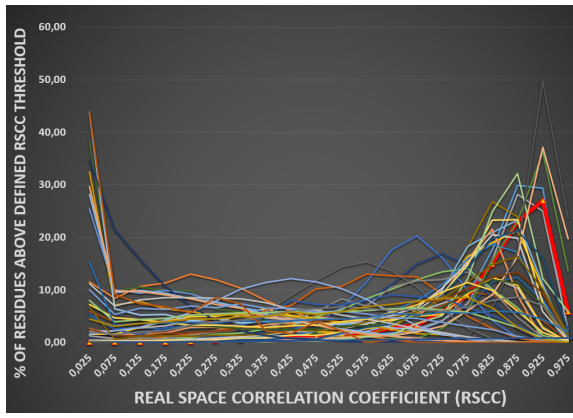

Figure 1. Histogram of RSCC of analyzed cryo-EM structures (5AFI structure is highlighted in red).

Keywords: cryo-EM, model fitting and refinement, validation 